\title{
Analysis of the Suzuki-Kasami Algorithm with SAL Model Checkers
}

\author{
Kazuhiro Ogata \\ NEC Software Hokuriku, Ltd. \\ ogatak@acm.org
}

\author{
Kokichi Futatsugi \\ School of Information Science, JAIST \\ kokichi@jaist.ac.jp
}

\begin{abstract}
We report on a case study in which SAL model checkers have been used to analyze the Suzuki-Kasami distributed mutual exclusion algorithm with respect to the mutual exclusion property and the lockout freedom property. SAL includes five different model checkers. In the case study, we have used two model checkers SMC (Symbolic Model Checker) and infBMC (infinite Bounded Model Checker). SMC has concluded that a finite-state model of the algorithm has the mutual exclusion property, but has found a counterexample to the lockout freedom property. The counterexample has led to one possible modification that makes the algorithm lockout free. We have also used infBMC to prove that an infinite-state model of the algorithm has the mutual exclusion property by $k$-induction.
\end{abstract}

Keywords: counterexamples, distributed algorithms, lockout freedom, model checkers, mutual exclusion.

\section{Introduction}

With the advance of computer hardware technology, efficient model checking algorithms[4] have been devised and then model checkers such as SMV[6] and SPIN[6] have been developed. Model checkers can be used to verify fully automatically that given systems have desired properties, provided that the systems should be modeled as finite-state machines. SAL $[1,2]$ is a toolkit for analyzing state machines, providing several tools. Unlike other existing model checkers, each of which usually implements one model checking algorithm, SAL includes five different model checkers. The five different model checkers are symbolic, bounded, infinite bounded, witness and explicit-state model checkers (SMC, BMC, infBMC, WMC and EMC, respectively). Users can select the most appropriate one among the five different model checkers for their problems.

The Suzuki-Kasami algorithm[9] is a distributed mutual exclusion algorithm. We have analyzed the algorithm in a semi-formal way[7] with respect to the mutual exclusion property and the lockout (starvation) freedom property. In the analysis, we have found a hidden assumption that is needed to make the algorithm lockout free. The assumption is that each node must try to enter its critical section infinitely often. In a distributed setting where some nodes try to enter their critical sections only finitely often, however, it is not guaranteed that the algorithm is lockout free.

In this paper, we report on a case study in which SAL model checkers have been used to analyze the SuzukiKasami algorithm with respect to the two properties. SMC has concluded that a finite-state model of the algorithm has the mutual exclusion property, but has found a counterexample to the lockout freedom property. The counterexample has led to one possible modification that makes the algorithm lockout free. The modification makes the algorithm lockout free even if some nodes try to enter their critical sections only finitely often. We have also used infBMC to prove that an infinite-state model of the algorithm has the mutual exclusion property by $k$-induction[3].

The rest of the paper is organized as follows. Section 2 outlines SAL. Section 3 describes the Suzuki-Kasami algorithm. Section 4 models the Suzuki-Kasami algorithm as a transition system. Section 5 specifies the transition system in SAL. Section 6 checks if a finite-state model of the Suzuki-Kasami algorithm has the mutual exlusion and lockout freedom properties with SMC. Section 7 verifies that an infinite-state model of the Suzuki-Kasami algorithm has the mutual exlusion property with infBMC by $k$-induction. Section 8 finally concludes the paper.

\section{SAL: Symbolic Analysis Laboratory}

SAL $[1,2]$ (see sal.csl.sri.com) provides the five different model checkers (SMC, BMC, infBMC, WMC and EMC). EMC is not provided by SAL 2.3, but will be included in a future release of SAL. SMC, BMC, WMC and EMC deal with state machines defined over finite data types, while infBMC can also handle infinite data types such as integers. SMC, BMC, infBMC and EMC checks if assertions written in LTL hold for state machines, while WMC can handle assertions in CTL. BMC uses a propositional SAT solver to find counterexamples no longer than 
some specified depth. It can also verify that finite state machines have LTL properties by $k$-induction[3]. By default, SAL uses ICS[5] (Integrated Canonizer and Solver; see www.icansolve.com) as its SAT solver. For infinite state machines, infBMC can do what BMC does.

The SAL language consists of a type system and four languages for expressions, transition relations, modules and contexts. The SAL's type system and expression language are similar to those of PVS[8] (see pvs.csl.sri.com). Transition relations can be specified using both guarded commands and SMV-style variable-wise invariants. A state machine is written as a module that can have parameters. A module basically consists of a section of variable declarations, an initialization section and a section of transition relations. Modules can be composed both synchronously and asynchronously to generate compound state machines. Contexts include type declarations, function definitions, modules and assertions. Contexts can have parameters that are type and variable declarations. The assertion language is not primitive in SAL but is defined in libraries associated with the model checker concerned.

\section{The Suzuki-Kasami Algorithm}

Let us consider a computer network consisting of a fixed number, say $N(\geq 1)$, of nodes. The nodes have no memory in common and can communicate only by exchanging messages. The distributed mutual exclusion problem is to solve a mutual exclusion requirement for such a computer network, namely to allow at most one node to stay in its critical section at any given moment. The Suzuki-Kasami algorithm[9] is a distributed algorithm solving the problem. The algorithm may be called SKDMXA in this paper. The basic idea in the algorithm is to transfer the privilege for entering the critical sections. Figure 1 shows the algorithm for node $i \in\{1,2, \ldots, N\}$ in a traditional style.

requesting and have privilege are Boolean variables. requesting indicates whether or not node $i$ either wants to enter or stays in its critical section, and have privilege indicates whether or not node $i$ owns the privilege. queue is a queue of integers denoting node identifiers. It contains identifiers of nodes that wait to enter their critical sections. $\ln$ and $r n$ are integer arrays of size $N . \ln [j]$ for each node $j \in\{1,2, \ldots, N\}$ is the sequence number of the node $j$ 's request granted most recently. $r n$ records the largest request number ever received from each one of the other nodes. Node $i$ uses $r n[i]$ to generate the sequence numbers of its own requests. For each node $i \in\{1,2, \ldots, N\}$, initially, requesting is false, have privilege is true if $i=1$ and false otherwise, queue is empty, and $\ln [j]$ and $r n[j]$ for each $j \in\{1,2, \ldots, N\}$ are 0 .

If node $i$ wants to enter its critical section, it first calls its own procedure $P 1$, which first sets requesting to true. If

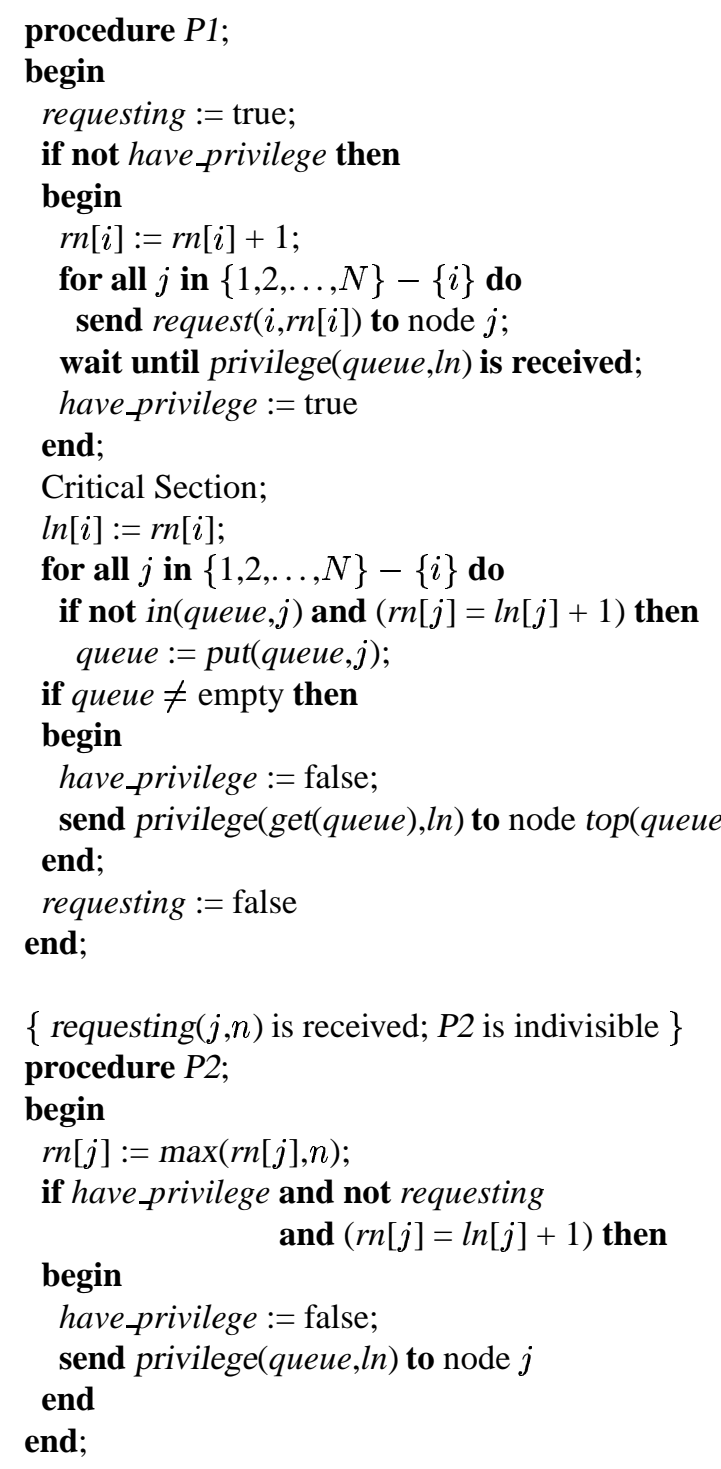

Figure 1. The Suzuki-Kasami algorithm

it happens to own the privilege, it immediately enters the critical section. Otherwise, it generates the next sequence number, namely, incrementing $r n[i]$, and sends the request message request $(i, r n[i])$ to all other nodes. When it receives a privilege message privilege (queue, $l n$ ), it enters the critical section. When it finishes executing the critical section, it sets $\ln [i]$ to its current sequence number $r n[i]$, indicating that the current request has been granted, and updates queue, namely that identifiers of nodes that want to enter their critical sections and are not in the queue yet are added to the queue. After that, if queue is not empty, node $i$ sets have privilege to false and sends the privilege message privilege (get(queue), ln) to the node found in the front of the queue, where get(queue) is the queue obtained by deleting 
the top element from queиe. Otherwise, node $i$ keeps the privilege. Finally node $i$ sets requesting to false and leaves procedure $P 1$.

Whenever request $(j, n)$ is delivered to node $i$, node $i$ executes its own procedure $P 2$. But, procedure $P 2$ has to be atomically executed. When node $i$ executes procedure $P 2$, it sets $r n[j]$ to $n$ if $n$ is greater than $r n[j]$. Then, if node $i$ owns the privilege, neither wants to enter nor stays in its critical section, and the $n$th request of node $j$ has not been granted, that is, $r n[j]=\ln [j]+1$, then it sets have privilege to false and sends the privilege message privilege(queue, $l n$ ) to node $j$.

\section{Modeling SKDMXA}

The algorithm is modeled as a transition system $\mathcal{S}_{\mathrm{SK}}^{L, M, N}$ that consists of a set $\mathcal{V}_{\mathrm{SK}}^{L, M, N}$ of variables, the initial condition $\mathcal{I}_{\mathrm{SK}}^{L, M, N}$ and a set $\mathcal{T}_{\mathrm{SK}}^{L, M, N}$ of transitions. The way of modeling the algorithm basically follows the one used in [7]. A transition may involve a condition called the effective condition. A transition can be executed only if the effective condition holds.

$\mathcal{V}_{\mathrm{SK}}^{L, M, N}$ includes requesting ${ }_{i}$ corresponding to requesting, have_privilege ${ }_{i}$ corresponding to have privilege, queue $_{i}$ corresponding to queue, $l n_{i}$ corresponding to $l n$, and $r n_{i}$ corresponding to $r n$, for each node $i \in$ $\{1,2, \ldots, N\} . \mathcal{V}_{\mathrm{SK}}^{L, M, N}$ also includes $i d x_{i}$ corresponding to $j, p c_{i}$, which indicates which part of the algorithm node $i$ is about to execute, and num_of_req ${ }_{i}$, which holds the number of requests node $i$ has made, for each node $i \in$ $\{1,2, \ldots, N\}$. In addition, $\mathcal{V}_{\mathrm{SK}}^{L, M, N}$ includes variables denoting the network. How to model the network in SAL will be described in the coming section.

Whenever node $i$ executes procedure $P 1$, the sequence number $r n[i]$ is incremented. Therefore, in order to make the state space of the transition system $\mathcal{S}_{\mathrm{SK}}^{L, M, N}$ finite, the number of requests made by each node $i$ should be finite, say $M(\geq 1)$.num_of_req $q_{i}$ is used to allow node $i$ to make at most $M$ requests.

$\mathcal{T}_{\mathrm{SK}}^{L, M, N}$ contains 13 transitions, which are $\operatorname{try}_{i}$, set_req $_{i}$, check_priv $_{i}$, inc_req_no $_{i}$, send_req $_{i}$, wait_priv $_{i}$, exit $_{i}$, complete_req $_{i}$, update_queue $_{i}$, check $_{-} q u e u e_{i}$, transfer_priv $_{i}$, reset_req $_{i}$ and receive_req ${ }_{i}$ for each node $i \in\{1,2, \ldots, N\}$. The transitions exhaustively and exclusively correspond to parts of the algorithm. The first 12 kinds of transitions are given labels (rem, 11, 12, 13, 14, 115, cs, $16,17,18,19$ and 110, respectively). $p c_{i}$ is set to one of the labels.

We describe (1) the effective condition of each transition and (2) how the transition changes variables in $\mathcal{V}_{\mathrm{SK}}^{L, M, N}$ when the transition is applied; variables that the transition does not change may not be described explicitly. For each variable $v, v$ and $v^{\prime}$ denote the values before and after a transition, respectively.

- $\operatorname{try}_{i}$ : (1) $p c_{i}=$ rem. (2) If num_of_req ${ }_{i}<M$, then $p c_{i}^{\prime}=11$ and num_of_req ${ }_{i}^{\prime}=n u m_{-} o f_{-} r e q_{i}+1$, and otherwise $p c_{i}^{\prime}=$ rem and num_of_req ${ }_{i}^{\prime}=n_{u} m_{-} o f_{-} r e q_{i}$.

- set_req $_{i}$ : (1) $p c_{i}=11$. (2) $p c_{i}^{\prime}=12$ and requesting $g_{i}^{\prime}=$ true.

- check_priv $_{i}:$ (1) $p c_{i}=12$. (2) If have_privilege ${ }_{i}=$ true, then $p c_{i}^{\prime}=\mathrm{cs}$, and otherwise $p c_{i}^{\prime}=13$.

- inc_req_no $o_{i}$ : (1) $p c_{i}=13$. (2) $p c_{i}^{\prime}=14, r n_{i}^{\prime}[i]=r n_{i}[i]+$ 1 and $i d x_{i}^{\prime}=1$.

- send_req $q_{i}$ : (1) $p c_{i}=14$. (2) If $i d x_{i}=N$, then $p c_{i}^{\prime}=15$ and $i d x_{i}^{\prime}=i d x_{i}$, and otherwise $p c_{i}^{\prime}=14$ and $i d x_{i}^{\prime}=i d x_{i}+$ 1. Besides, a request message with $i$ and $r n_{i}[i]$ is sent to node $i d x_{i}$ (is put into the network) unless $i d x_{i}=i$.

- wait_priv $_{i}$ : (1) $p c_{i}=15$ and there exists a privilege message with a queue $Q$ of integers and an integer array $L N$ of size $N$, whose destination is node $i$, in the network. (2) $p c_{i}^{\prime}=\mathrm{cs}$, have_privilege ${ }_{i}^{\prime}=$ true, queue $_{i}^{\prime}=Q$ and $l n_{i}^{\prime}=L N$. Besides, the privilege message is removed from the network.

- exit $_{i}$ : (1) $p c_{i}=\mathrm{cs}$. (2) $p c_{i}^{\prime}=16$.

- complete_req $_{i}$ : (1) $p c_{i}=16$. (2) $p c_{i}^{\prime}=17, \ln _{i}^{\prime}[i]=r n_{i}[i]$ and $i d x_{i}^{\prime}=1$.

- update $_{-} q u e u e_{i}:$ (1) $p c_{i}=17$. (2) If $i d x_{i}=N$ then $p c_{i}^{\prime}=18$ and $i d x_{i}^{\prime}=i d x_{i}$, and otherwise $p c_{i}^{\prime}=17$ and $i d x_{i}^{\prime}=i d x_{i}+1$. If $r n_{i}\left[i d x_{i}\right]=\ln _{i}\left[i d x_{i}\right]+1$ and $i d x_{i}$ is not in queue $_{i}$, then queue $e_{i}^{\prime}=\operatorname{put}\left(q u e u e_{i}, i d x_{i}\right)$, and otherwise queue $_{i}^{\prime}=$ queue $_{i}$.

- check_queue $_{i}:$ (1) $p c_{i}=18$. (2) If queue is empty, then $p c_{i}^{\prime}=110$, and otherwise $p c_{i}^{\prime}=19$.

- transfer_priv $_{i}$ : (1) $p c_{i}=19$. (2) $p c_{i}^{\prime}=110$ and have_privilege $_{i}^{\prime}=$ false. Besides, the privilege message with get $\left(q u e u e_{i}\right)$ and $l_{i}$ is sent to node top $\left(q u e u e_{i}\right)$.

- reset_req $_{i}:$ (1) $p c_{i}=110$. (2) $p c_{i}^{\prime}=$ rem and requesting ${ }_{i}^{\prime}=$ false.

- receive_req $_{i}$ : (1) There exists a request message with $j(\neq i)$ and $n$ in the network, whose destination is node $i$. (2) $r n_{i}^{\prime}[j]=\max \left(r n_{i}[j], n\right)$. Let $C$ be have_privilege ${ }_{i} \wedge$ $\neg$ requesting ${ }_{i} \wedge r n_{i}^{\prime}[j]=l n_{i}[j]+1$. If $C$ is true, then have_privilege ${ }_{i}^{\prime}=$ false and the privilege message with queue $e_{i}$ and $l n_{i}$ is sent to node $n$, and otherwise have_privilege $_{i}^{\prime}=$ have_privilege $_{i}$. Besides, the request message is removed from the network.

$\mathcal{I}_{\mathrm{SK}}^{L, M, N}$ is that requesting $i_{i}$ is false, have_privilege ${ }_{i}$ is true if $i=1$ and false otherwise, queue ${ }_{i}$ is empty, each element of $l n_{i}$ is 0 , each element of $r n_{i}$ is $0, i d x_{i}$ is $1, p c_{i}$ is rem and num_of_req $q_{i}$ is 0 for each $i \in\{1,2, \ldots, N\}$. In addition, variables denoting the network are set to values denoting the empty network. 


\section{Specification of $\mathcal{S}_{\mathrm{SK}}^{L, M, N}$ in SAL}

We describe how to specify $\mathcal{S}_{\mathrm{SK}}^{L, M, N}$ in SAL, specifically how to encode queues in more basic types, how to model the network and how to specify the behavior of each node.

\subsection{Encoding Queues}

Since recursive data types cannot be used in SAL specifications to be model checked, queues should be encoded in more basic types. Arrays and integers are used to encode queues. Type Queue is declared as a record type whose fields are data and $t l$ as follows:

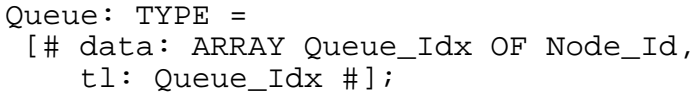

Types Node_Id and Queue_Idx are declared as bounded integers $[1 \ldots \mathrm{N}]$ and $[0 \ldots(\mathrm{L}+1)]$, respectively. $\mathrm{N}$ is the number of nodes involved in the algorithm and $\mathrm{L}$ is the capacity of each queue.

Given an instance $q$ of Queue, the $L$ spaces from q.data [1] to q.data [L] are used to store queue elements; q. data [1] is always the top element of the queue if the queue is not empty; $q . t l$ points the space where an element will be put next if the queue is not full and therefore $q$. data [q.tl - 1] is always the last element of the queue if the queue is not empty. We declare 9 functions related to Queue, which are new_empty_queue returning the empty queue, full? checking if a given queue is full, empty? checking if a given queue is empty, in? checking if a given queue includes a given element, in_aux? that is an auxiliary function for in?, top returning the top element of a given queue, put returning the queue obtained by putting a given element into a given queue at the end, get returning the queue obtained by deleting the top element of a given queue, and get_aux that is an auxiliary function for get.

In this paper, we only show the definitions of in_aux?, in?, get_aux and get:

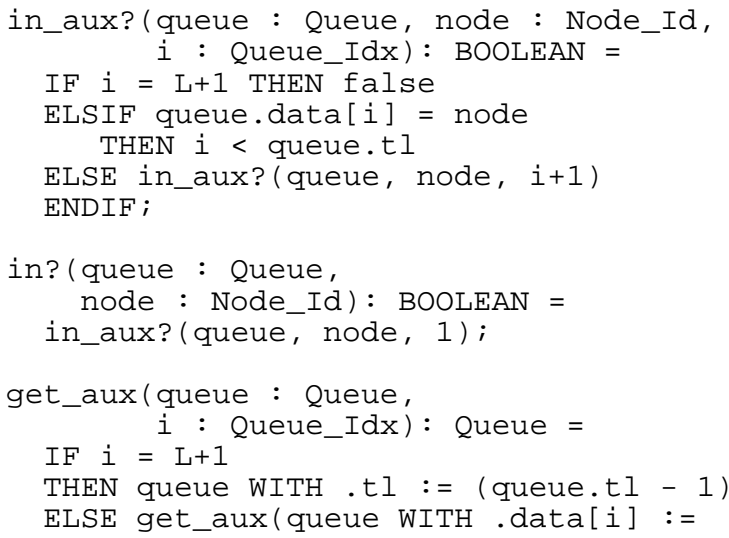

ENDIF ;

get (queue : Queue): Queue =

IF empty? (queue)

THEN queue ELSE get_aux (queue, 1) ENDIF;

Given an array $a$, an index $i$ and a value $v$, the expression $a$ WITH $. i:=v$ denotes the array obtained by assigning $v$ to the $i$ th place of $a$.

In order to use a function in a SAL specification to be model checked, SAL must be able to prove the function terminating. If a function has a conditional choice (IF THEN ELSE ENDIF) statement whose condition is $L=R$ such that both $L$ and $R$ include variables whose values can be determined only at runtime, then SAL cannot be able to prove the function terminating. Therefore, SAL cannot prove the following definition of in_aux? (which seems more straightforward than that shown above) terminating:

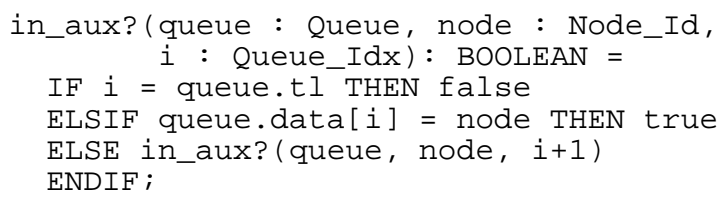

In the first definition of in_aux?, since the value of $L$ is given in advance and is not mutable, the value of $L+1$ can be determined statically.

\subsection{Modeling the Network}

For each kind of message and each ordered $(i, j)$ pair of nodes, we use a cell with which node $i$ sends to node $j$ one message of this kind. For each kind of message, we then use an array whose elements are arrays of messages of this kind. Since there are two kinds of messages, request and privilege messages, we use two such arrays to model the network.

In a module called node where the behavior of each node is written, we use two global variables reqmedium and privmedium to denote the network. The type of reqmedium is

ARRAY Node_Id OF ARRAY Node_Id OF ReqMsg

reqmedium[i][j] denotes the cell with which node $i$ sends a request message to node $j$. The type of privmedium is

ARRAY Node_Id OF ARRAY Node_Id OF PrivMsg

privmedium [i] [ j] denotes the cell with which node $i$ sends a privilege message to node $j$.

Types ReqMsg and PrivMsg denote cells with which messages are transferred. ReqMsg is declared as follows:

[\# new: BOOLEAN, req: Request \#]

PrivMsg is declared as follows: 


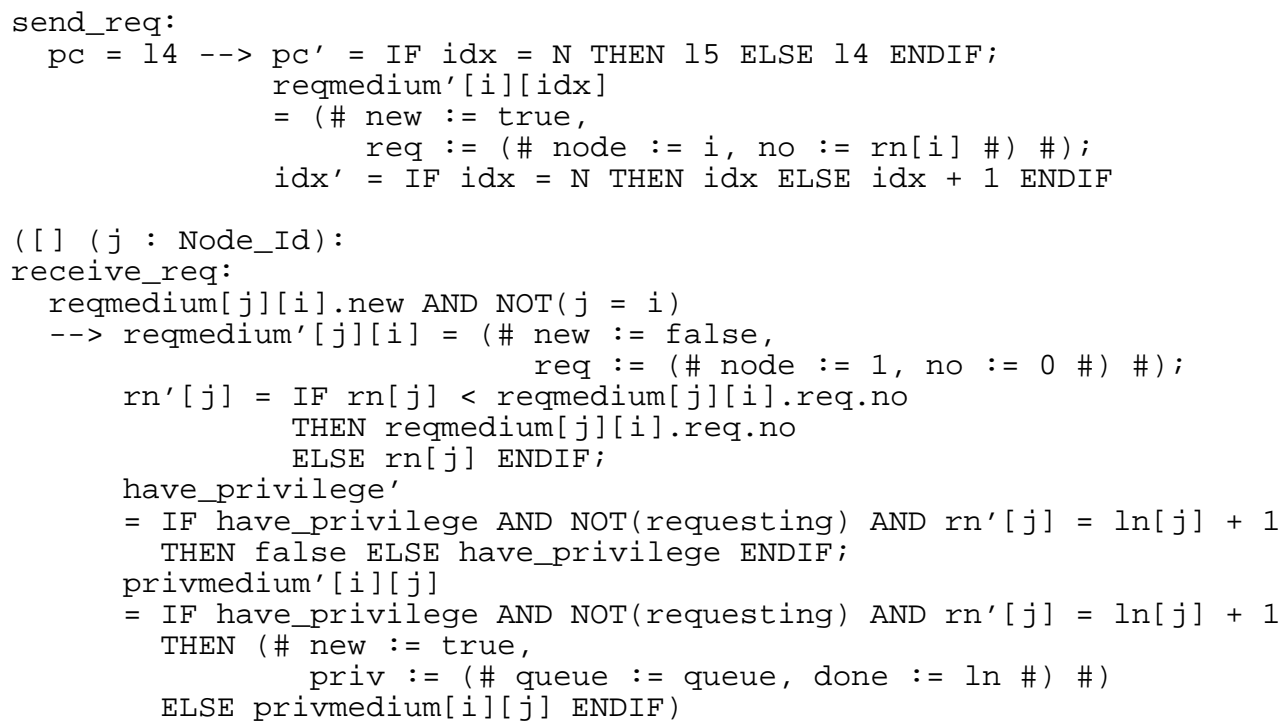

Figure 2. Guarded commands corresponding to send_req $_{i}$ and receive_req $q_{i}$

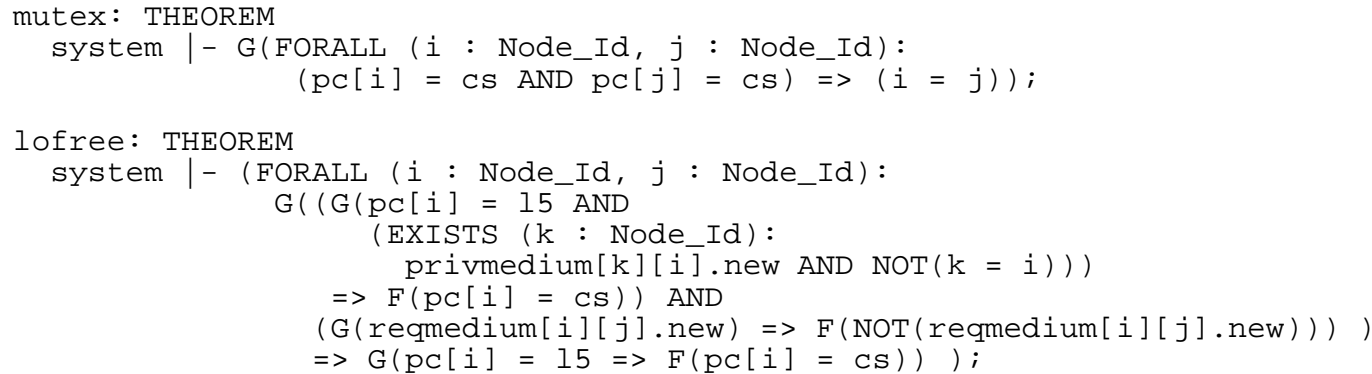

Figure 3. Assertions for the mutual exclusion and lockout freedom properties

[\# new: BOOLEAN, priv: Privilege \#]

For each record type, the second field holds a message to be transferred, and the first field indicates whether or not the cell contains a message.

Types Request and Privilege denote request and privilege messages, respectively. Request is declared as

[\# node: Node_Id, no: Bnat \#]

Privilege is declared as

[\# queue: Queue, done: ARRAY Node_Id OF Bnat \#]

Type Bnat is $[0 \ldots \mathrm{M}]$ and $\mathrm{M}$ is the number of requests made by each node.

\subsection{Specifying the Behavior of each Node}

The behavior of each node is written in module node, which has a parameter $i$ whose type is Node_Id. In addition to the two global variables representing the network, namely reqmedium and privmedium, the following local variables are declared: requesting whose type is BOOLEAN, have_privilege whose type is BOOLEAN, $r n$ whose type is ARRAY Node_Id OF Bnat, In whose type is ARRAY Node_Id OF Bnat, queue whose type is Queue, idx whose type is Node_Id, pc whose type is Label, where Type Label is an enumeration type whose values correspond to the 12 labels such as rem and 11, and num_of_req whose type is Bnat. The variables correspond to requesting $_{i}$, have_privilege ${ }_{i}, r n_{i}, \ln _{i}$, queue $_{i}$, $i d x_{i}, p c_{i}$ and $n u m_{-} o f \_r e q$ of transition system $\mathcal{S}_{\mathrm{SK}}^{L, M, N}$ described in Sect.4, respectively. The variables are initialized as described in Sect.4.

The 13 transitions described in Sect.4 are written using guarded commands. A guarded command is in the form Label: Guard --> Effect, where Label is the label given to the guarded command, which is an option, Guard is the condition, of the guard of the guarded command, and Effect is the effects (i.e. how to change the values of variables) 
of the guarded command. The guarded commands corresponding to the 13 transitions are asynchronously composed to specify the behavior of each node as follows

$$
\begin{gathered}
\text { Label }_{1}: \text { Guard }_{1}->>\text { Effect }_{1} \\
\text { [] } \text { Label }_{2}: \text { Guard }_{2}->>\text { Effect }_{2} \\
\ldots \\
\text { [] } \text { Label }_{13}: \text { Guard }_{13}->\text { Effect }_{13}
\end{gathered}
$$

Some transition is written as multiple guarded commands that are asynchronously composed as follows:

$$
\left([](j: T): L: G_{j}->E_{j}\right),
$$

which is equivalent to

$$
L: G_{1}->E_{1}[] \ldots[] L: G_{N}->E_{N},
$$

where $T$ is $[1 \ldots N]$.

Figure 2 shows the guarded commands corresponding to send_req $_{i}$ and receive_req ${ }_{i}$. receive_req ${ }_{i}$ is written as $\mathrm{N}$ guarded commands. The remaining transitions are written using guarded commands likewise.

Instances of module node are asynchronously composed to obtain the SAL specification of $\mathcal{S}_{\mathrm{SK}}^{L, M, N}$, which is written as follows:

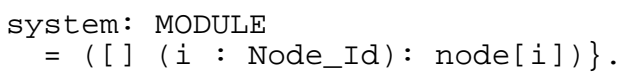

\section{Model Checking $\mathcal{S}_{\mathrm{SK}}^{2,2,2}$}

By having each of N, L and M fixed, say 2, we use SMC to check if $\mathcal{S}_{\mathrm{SK}}^{2,2,2}$ has the mutual exclusion property and the lockout freedom property. The properties are written as the assertions labeled mutex and lof ree, respectively, shown in Fig. 3. G and F are LTL temporal operators Henceforth (Always) and Eventually, respectively. We use the assumption that weak fairness is given to each of wait_priv and receive_req transitions to check the lockout freedom property. SMC concludes that mutex holds for $\mathcal{S}_{\mathrm{SK}}^{2,2,2}$, namely system such that $L=2, M=2$ and $N=2$, but presents a counterexample to lof ree.

A counterexample generated by a SAL model checker is composed of two lists $P$ and $L$ of pairs, each of which consists of a state (assignments to system variables) and the label of the guarded command applied to reach the state of the next pair. The next of the last pair of $P$ is the first of $L$ and the next of the last pair of $L$ is the first of $L$. $P$ corresponds to a finite path beginning in an initial state and leading to a loop represented by $L$. The loop represents a state or a transition sequence that violates a given formula.

Figure 4 shows an excerpted counterexample to the lockout freedom generated by SMC. The counterexample says

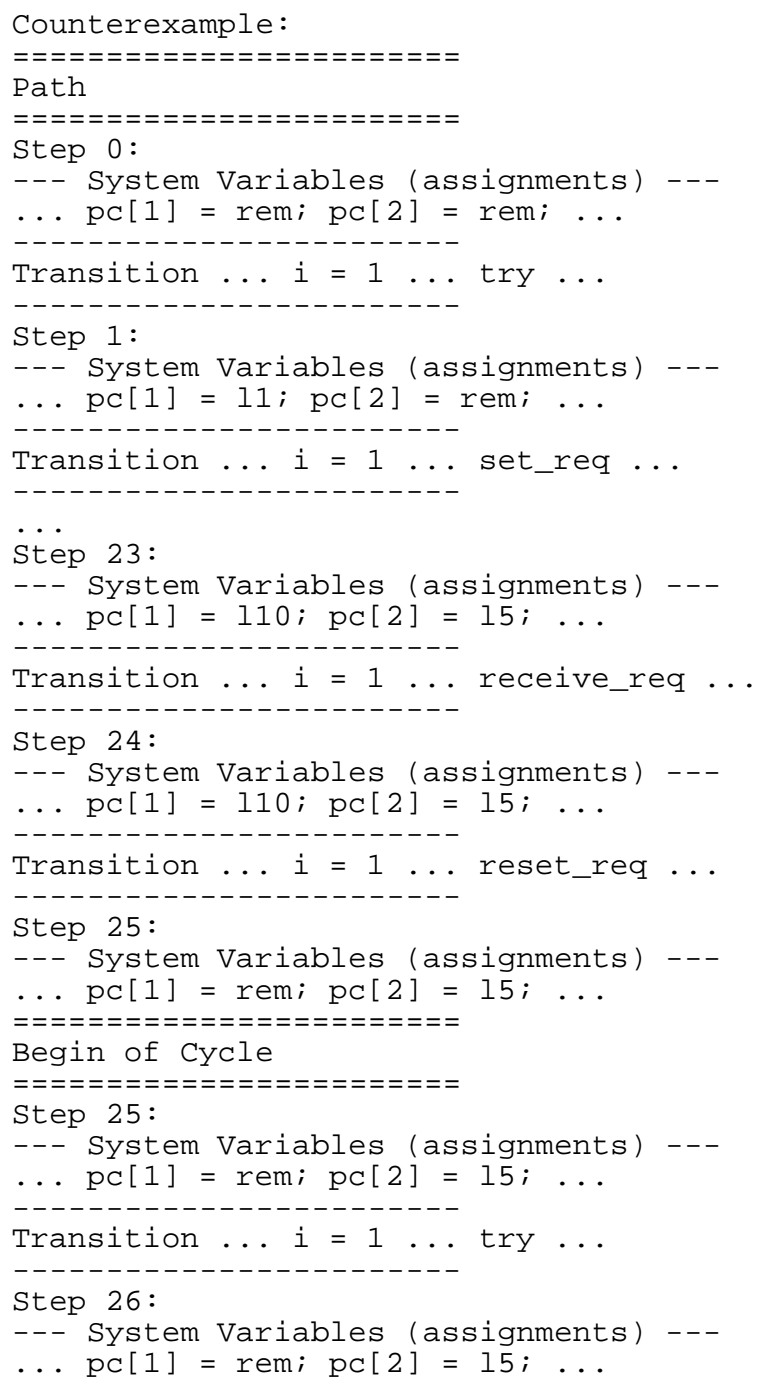

\section{Figure 4. An excerpted counterexample to the lockout freedom}

that if node 1 receives a request message from node 2 at 110 , then node 2 may have to wait for a privilege message forever at 15 . When node 1 receives a request message from node 2 at 110 , node 1 does not transfer the privilege to node 2 because its requesting is true. If node 1 will not execute procedure $P 1$ anymore, node 1 will never transfer the privilege to node 2 and node 2 will wait for a privilege message forever.

The counterexample suggests that each node should not receive any request messages at 110 . Therefore, the condition NOT $(\mathrm{pc}=110)$ is added to the guard of receive. But this is not sufficient. Two more performances of model checking have us notice that NOT $(\mathrm{pc}=18)$ and NOT $(\mathrm{pc}=17)$ should also be added to the guard. SMC 


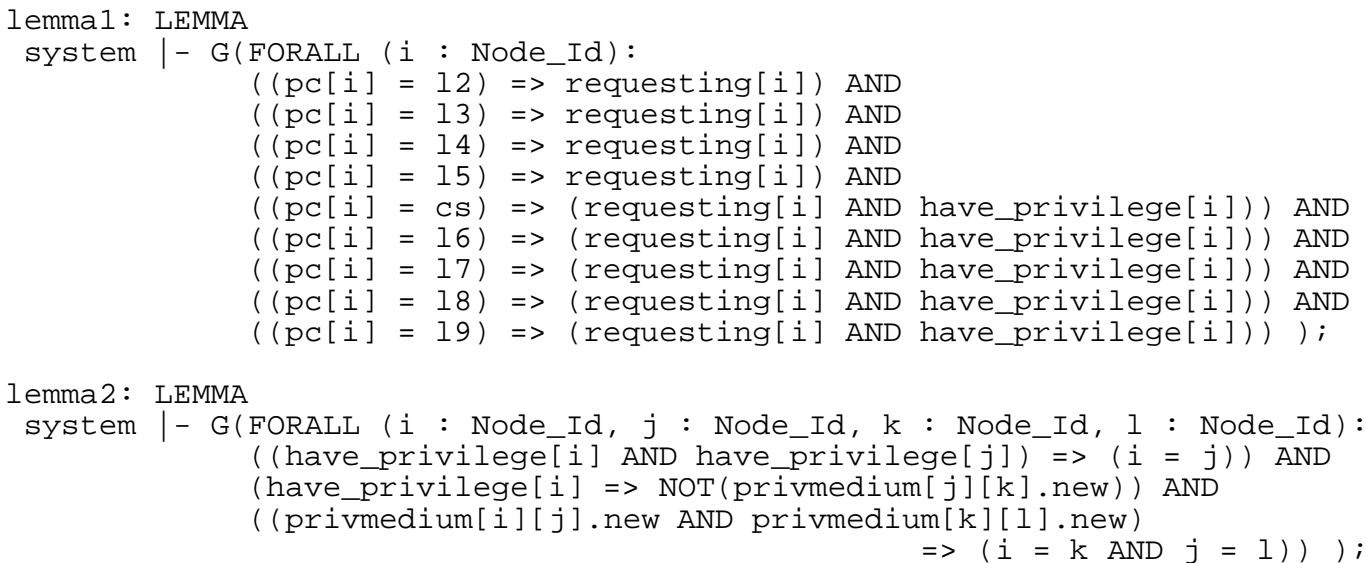

\section{Figure 5. Lemmas}

finally concludes that both mutex and lofree hold for the revised $\mathcal{S}_{\mathrm{SK}}^{2,2,2}$.

\section{Verification of $\mathcal{S}_{\mathrm{SK}}^{2, \infty, 2}$ with $k$-induction}

In $\mathcal{S}_{\mathrm{SK}}^{L, M, N}$ analyzed with SMC, we made the number $M$ of requests made by each node finite to make the state space of $\mathcal{S}_{\mathrm{SK}}^{L, M, N}$ finite. We relax the restriction, namely allowing each node to make an arbitrary number of requests. Let such $\mathcal{S}_{\mathrm{SK}}^{L, M, N}$ is represented by $\mathcal{S}_{\mathrm{SK}}^{L, \infty, N}$. infBMC can be used to analyze $\mathcal{S}_{\mathrm{SK}}^{L, \infty, N}$ whose (even reachable) state space is infinite. Let both of $L$ and $N$ be 2 .

The property to be analyzed is the mutual exclusion property. We can use infBMC to search for a counterexample to mutex no longer than some specified depth, say 10. infBMC concludes that there are no counterexamples in the specified range.

We can also use infBMC to verify that $\mathcal{S}_{\mathrm{SK}}^{2, \infty, 2}$ has the mutual exclusion property with $k$-induction. To this end, first we should verify that two lemmas hold for $\mathcal{S}_{\mathrm{SK}}^{2, \infty, 2}$. The first lemma is written as the assertion labeled lemma 1 shown in Fig.5. infBMC can verify that lemma1 holds for $\mathcal{S}_{\mathrm{SK}}^{2, \infty, 2}$ with 1-induction. The second lemma is written as the assertion labeled lemma2 shown in Fig.5. infBMC can verify that lemma 2 holds for $\mathcal{S}_{\mathrm{SK}}^{2, \infty, 2}$ with 1 -induction using lemma 1 as a lemma. Using the two lemmas, infBMC can verify that mutex holds for $\mathcal{S}_{\mathrm{SK}}^{2, \infty, 2}$ with 1-induction.

The structure of the verification with $k$-induction would be useful when we verify that the infinite model involving an arbitrary number of nodes has the mutual exclusion property with a theorem prover such as PVS[8]. In other words, we would need the two lemmas to verify that $\mathcal{S}_{\mathrm{SK}}^{L, \infty, N}$ involving an arbitrary number $N$ of nodes has the property. Actually, when we verified it in a semi-formal way[7], we used two lemmas that were almost the same as lemma 1 and lemma2.

\section{Conclusion}

We reported on a case study in which SAL model checkers, precisely SMC and infBMC, have been used to analyze the Suzuki-Kasami algorithm with respect to the mutual exclusion property and the lockout freedom property. In the case study, we have found out one possible modification that makes the algorithm lockout free even if some nodes try to enter their critical sections only finitely often.

\section{References}

[1] L. de Moura, S. Owre, H. Rueß, J. Rushby, N. Shankar, M. Sorea, and A. Tiwari. SAL 2. In 16th CAV, LNCS 3114, pages 496-500. Springer, 2004.

[2] L. de Moura, S. Owre, and N. Shankar. The SAL language manual. CSL Technical Report SRI-CSL-01-02 (Rev. 2), August 2003.

[3] L. de Moura, H. Rueß, and M. Sorea. Bounded model checking and induction: From refutation to verifi cation. In 15th $C A V$, LNCS 2392, pages 14-26. Springer, 2003.

[4] J. Edmund M. Clarke, O. Grumberg, and D. A. Peled. Model Checking. The MIT Press, 2001.

[5] J.-C. Filliâtre, S. Owre, H. Rueß, and N. Shankar. ICS: integrated canonizer and solver. In 13th CAV, LNCS 2102, pages 246-249. Springer, 2001.

[6] G. J. Holzmann. The SPIN Model Checker-Primer and Reference Manual. Addison-Wesley, 2004.

[7] K. Ogata and K. Futatsugi. Formal analysis of Suzuki\&Kasami distributed mutual exclusion algorithm. In 5th FMOODS, pages 181-195. Kluwer, 2002.

[8] S. Owre, J. M. Rushby, and N. Shankar. PVS: A prototype verifi cation system. In 11th CADE, LNAI 607, pages 748752. Springer, 1992.

[9] I. Suzuki and T. Kasami. A distributed mutual exclusion algorithm. ACM TOCS, 3(4):344-349, 1985. 